\title{
Evaluación de la Responsabilidad Social Universitaria: una revisión sistemática de literatura
}

\author{
Evaluation of University Social Responsibility: A Systematic Literature Review
}

\author{
Andrés Rodrigo Santana Murcia \\ Universidad Nacional de Colombia \\ Bogotá, Colombia
}

\begin{abstract}
Resumen:
La Responsabilidad Social Universitaria (RSU) se ha consolidado en las últimas décadas, como el paradigma de la gestión universitaria del siglo XXI (Vallaeys, 2014; Coppa, 2011; Villar, 2009). Numerosos trabajos han intentado hacer una aproximación a una definición general; otros, han analizado la implementación de iniciativas de RSU en distintos contextos, como un aporte a su interpretación y estudio; y algunos más han propuesto esquemas de evaluación de las percepciones y prácticas de RSU en Instituciones de Educación Superior (IES) de diversas latitudes. El presente trabajo examina los avances en la evaluación de la RSU, dada su importancia para el autodiagnóstico y la generación de procesos de mejora continua en las funciones misionales de formación, investigación, internacionalización y extensión. A partir de la revisión sistemática de literatura (RSL) y el análisis de contenido, se presenta un resumen de las publicaciones más relevantes, resaltando los puntos de encuentro más representativos. Se identificaron como principales tendencias el uso de cuestionarios de percepción, la adaptación de los indicadores del GRI y el análisis de memorias, páginas web e informes de gestión. Finalmente, se ofrecen conclusiones acerca del estado del conocimiento y algunas recomendaciones para el desarrollo de nuevas investigaciones en este campo.
\end{abstract}

Palabras clave: Responsabilidad Social Universitaria; evaluación; autodiagnóstico; convivencia; estrategias de aprendizaje.

\begin{abstract}
:
University Social Responsibility (USR) has been consolidated in recent decades as the management's paradigm of XXI the century university (Vallaeys, 2014; Coppa, 2011; Villar, 2009). There are many works that have tried to approximate a general definition; others have analyzed the implementation of RSU initiatives in different contexts, as a contribution to their interpretation and study; and some more have proposed evaluation schemes of the perceptions and practices of USR in Institutions of Higher Education (HEI) of different latitudes. This paper examines the advances in the evaluation of USR because of its importance for self-diagnosis and the generation of continuous improvement processes in the missionary functions of education, research, internationalization, and extension. Based on the systematic literature review (SLR) and content analysis, a summary of the most relevant publications is presented, highlighting the most representative meeting points. There were identified as main trends the use of perception questionnaires, the adaptation of the GRI indicators and the analysis of reports, web pages and management reports. Finally, conclusions are offered about the state of knowledge and some recommendations for the development of new research in this field.
\end{abstract}

Keywords: University Social Responsibility; assessment; auto diagnosis; coexistence; learning strategies.

Pag. 16-31
Publicado:

05/01/2021 


\section{Evaluación de la Responsabilidad Social Universitaria: una revisión sistemática de literatura.}

\section{| Introducción}

La Responsabilidad Social Empresarial (RSE) es uno de los conceptos que más rápidamente se ha extendido a nivel global durante las últimas décadas (Amato et al., 2016; De la Cuesta González, 2004; Martínez \& Soza, 2012; Nieto Antolín \& Fernández Gago, 2004; Raufflet et al., 2012). Aunque no existe un consenso sobre su significado (Martínez García de Leaniz \& Rodríguez del Bosque Rodríguez, 2013; Crespo Razeg, 2010; Votaw, 1972), frecuentemente se ha relacionado con un actuar ético de las organizaciones y la integración voluntaria en la gestión de preocupaciones sociales, económicas y ambientales (Comisión de las Comunidades Europeas, 2002; Correa et al., 2004; Eberhard-Harribey, 2006; Logsdon, 2004; Wheeler et al., 2003; World Business Council for Sustainable Development [WBCSD] \& United Nations Environment Programme Finance Initiative [UNEP FI], 2004).

Si bien, esta visión es aplicable a compañías de distintos sectores y tipos, existen actividades e instituciones sobre las cuales se ejercen mayores presiones sociales, dada su naturaleza y misión. Tal es el caso de las Universidades e Instituciones de Educación Superior (en adelante IES), entes encargados de prestar un servicio público que cumple con una función social.

En esencia, la responsabilidad social de las IES de la denominada sociedad del conocimiento debe centrarse en la reflexión acerca de la efectividad e impacto que sus acciones tienen sobre ella (Gaete Quezada, 2016b; Organización de las Naciones Unidas para la Educación, la Ciencia y la Cultura [UNESCO], 1998), la comprensión de los problemas polifacéticos que la afectan, sus distintas dimensiones -sociales, económicas científicas y culturales- y su capacidad para hacerles frente
(Organización de las Naciones Unidas para la Educación, la Ciencia y la Cultura [UNESCO], 2009).

En un contexto en el que la hegemonía, legitimidad e institucionalidad de la Universidad se han visto amenazadas por su incapacidad para (1) desarrollar simultáneamente funciones contradictorias - producir pensamiento crítico y conocimiento científico, por un lado, y patrones culturales y conocimientos instrumentales para el sistema capitalista, por el otro-, (2) crear consenso entre la jerarquización de los saberes especializados y la democratización de la universidad y (3) enfrentar la dicotomía entre la reivindicación de la autonomía universitaria y la eficiencia y productividad empresarial (De Sousa Santos, 2007), la RSU aparece como una solución viable al conjugar la búsqueda de soluciones factibles a las demandas de la sociedad (Viteri-Moya et al., 2012) con la gestión ética de la calidad universitaria (Ramallo, 2015).

Por ello, la RSU puede definirse como la gestión integral y estratégica de los impactos que las funciones sustantivas de formación, investigación y proyección social generan sobre los grupos de interés de la universidad, con miras a promover la justicia social y el desarrollo humano sostenible, al tiempo que se da respuesta a las demandas propias de la sociedad del conocimiento.

La importancia de la evaluación de la Responsabilidad Social (RS) subyace al hecho de que una auditoría social, basada en estándares e indicadores que traduzcan los principios de la RS en variables medibles, permite llegar a concluir si una organización es o no socialmente responsable (De la Cuesta González \& Valor Martínez, 2003), es decir, si cuenta con un sistema de gestión que asume el conjunto de obligaciones y compromisos legales 
y éticos adquiridos con sus grupos de interés (De la Cuesta González \& Valor Martínez, 2003) o, si por el contrario, las estrategias de RS se plantean como un área periférica a la gestión (Caro González \& Castellanos Verdugo, 2007).

La evaluación de la RSU cobra una relevancia aún mayor, al permitir a la sociedad y sus instituciones conocer el grado en que las IES asumen sus compromisos como entes generadores de nuevos conocimientos y de profesionales capaces de llevar a cabo las transformaciones sociales, políticas y económicas que demanda la sociedad, en procura de un desarrollo sostenible (Aristimuño et al., 2011). Temas como la pobreza, la desintegración social y la protección de los recursos naturales deberían estar en el centro de las preocupaciones de los egresados de los distintos programas de formación profesional (Jiménez et al., 2004), lo que conlleva a que estas instituciones, sin importar si son de naturaleza pública o privada, deban comprometerse con acciones de formación integral y otras funciones que fortalezcan y justifiquen la aplicación de su responsabilidad social más allá de las actividades de extensión, investigación, gestión y proyección social (Torres Castaño \& Sánchez Vásquez, 2014).

Así mismo, un autodiagnóstico basado en el uso de indicadores objetivos que sirvan como herramientas para la evaluación de los resultados de la RSU (Gaete Quezada, 2016a; Rodríguez Fernández, 2010; Zárate Rueda \& García Rincón, 2014; Vallaeys et al., 2009) y ofrezcan apoyo a la toma de decisiones (Lozano, 2006) promueve la mejora continua, la actuación y el aprendizaje de la Universidad en los ámbitos organizacional, educativo, social y de conocimiento (Londoño Franco, 2013).

Algunos de los problemas que aparecen con frecuencia a la hora de evaluar el compromiso social de las universidades son: (1) la variedad de enfoques adoptados, (2) la falta de atención a los resultados e impactos y (3) la ausencia de instrumentos y herramientas estandarizadas (Hart \& Northmore, 2010).
Instituciones como el Banco Interamericano de Desarrollo [BID] (Vallaeys et al., 2009), la Unión de Responsabilidad Social Universitaria Latinoamericana [URSULA] (Unión de Responsabilidad Social Universitaria Latinoamericana [URSULA], 2018), el Observatorio Regional de Responsabilidad Social para América Latina y el Caribe [ORSALC] (Grimaldo et al., 2018) y la Asociación de Universidades confiadas a la Compañía de Jesús en América Latina [AUSJAL] (Asociación de Universidades confiadas a la Compañía de Jesús en América Latina [AUSJAL]-Red, 2009) han hecho esfuerzos importantes por generar indicadores generales, escalas y sistemas de evaluación de la Responsabilidad Social Universitaria que permitan superar estas dificultades.

Esta investigación hace un recorrido por los trabajos empíricos dirigidos a crear esquemas de evaluación de la RSU en IES de diferentes tipologías. Para lograrlo se realizó una revisión sistemática de literatura (RSL) en la base de datos Scopus, seleccionada por el volumen y tipo de publicaciones con las que cuenta; el grado de actualización de los resultados que arroja y las herramientas de análisis bibliométrico que incluye. Los resultados obtenidos se depuraron tras un examen de títulos y palabras clave. Se trabajaron aquellos documentos cuyos títulos se vincularán directamente con los términos: Responsabilidad Social, Responsabilidad Social Universitaria (RSU), Responsabilidad Social en Universidades, Responsabilidad Social en Instituciones de Educación Superior y evaluación de la Responsabilidad Social Universitaria.

El trabajo de revisión sistemática y análisis bibliométrico de Duque y Cervantes-Cervantes (2019) sirvió como insumo para identificar una serie de categorías de clasificación y contrastarlas con los resultados obtenidos en la RSL.

El documento se divide en tres grandes secciones. En la primera parte se expone una descripción de la metodología 
empleada, las ecuaciones de búsqueda utilizadas, los hallazgos iniciales y el método de depuración adelantado. La segunda sección: "la evaluación de la RSU" muestra los resultados de la RSL y ordena las publicaciones cronológicamente, agregando un análisis de los esquemas de evaluación identificados.

La sección final exhibe las conclusiones de la RSL, junto con una descripción de sus alcances y limitaciones con el ánimo de aportar al avance del conocimiento en este ramo y ofrecer una guía para el planteamiento de interrogantes que orienten futuras investigaciones.

\section{| Método}

La RSL se define como un método sistemático diseñado para identificar, evaluar e interpretar el trabajo de los investigadores y académicos de un campo específico (Fink, 1998). Su objetivo es el de recopilar y analizar críticamente los estudios y trabajos de investigación más relevantes sobre un tema de interés (García-Peñalvo, 2017) con el objeto de establecer lagunas en el estado de conocimiento y con ello necesidades de investigación (Maier, 2013).

Se ha escogido este método debido a que permite realizar un resumen exhaustivo de la literatura disponible sobre un tema o pregunta de investigación (García-Peñalvo, 2017), al tiempo que posibilita a los lectores replicar el proceso y verificar los resultados obtenidos (Sáenz, 2001).

La presente investigación es de corte cualitativo, toda vez que se propone analizar críticamente las publicaciones existentes sobre evaluación de la RSU en este siglo. Con ello se busca identificar las tendencias generales en este tipo de estudios y determinar los aspectos evaluados, a fin de establecer posibles vacíos y proponer nuevos elementos de análisis.

Las RSL, a diferencia de otras revisiones, como las narrativas, cuentan con una serie de características que han sido resumidas por Ferreras-Fernández et al. (2016) de la siguiente forma: (a) Objetivos claramente establecidos con criterios de elegibilidad predefinidos, (b) Metodología explícita y reproducible, (c) Búsqueda sistemática que intenta identificar todos los estudios que cumplen criterios de elegibilidad, (d) Evaluación de la validez de los hallazgos, y (e) Presentación y síntesis sistemática de las características y hallazgos.

Los objetivos perseguidos, la metodología empleada, los resultados esperados y los ejes de trabajo de las conclusiones se muestran en la figura 1.

\section{Figura 1}

Componentes de la RSL

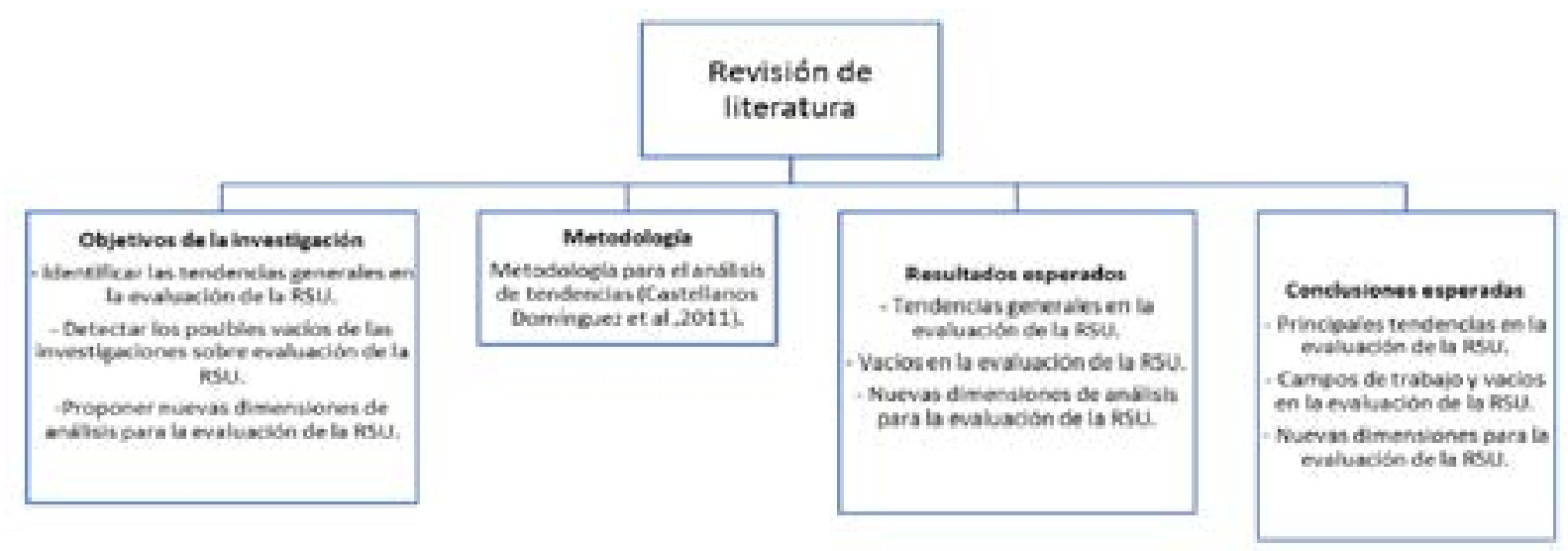

Nota: Componentes de la RSL definidos por Maier (2013) y su adaptación al presente estudio. 
Se seleccionó como metodología para la recopilación y análisis de la información los lineamientos metodológicos para el análisis de tendencias de Castellanos Domínguez et al. (2011), debido a su afinidad con los planteamientos de Ferreras-Fernández et al. (2016) y su demostrada utilidad en investigaciones de similares características. La figura 2 presenta las fases del proceso.

\section{Figura 2}

Esquema metodológico para el análisis de tendencias Castellanos Domínguez et al. (2011)

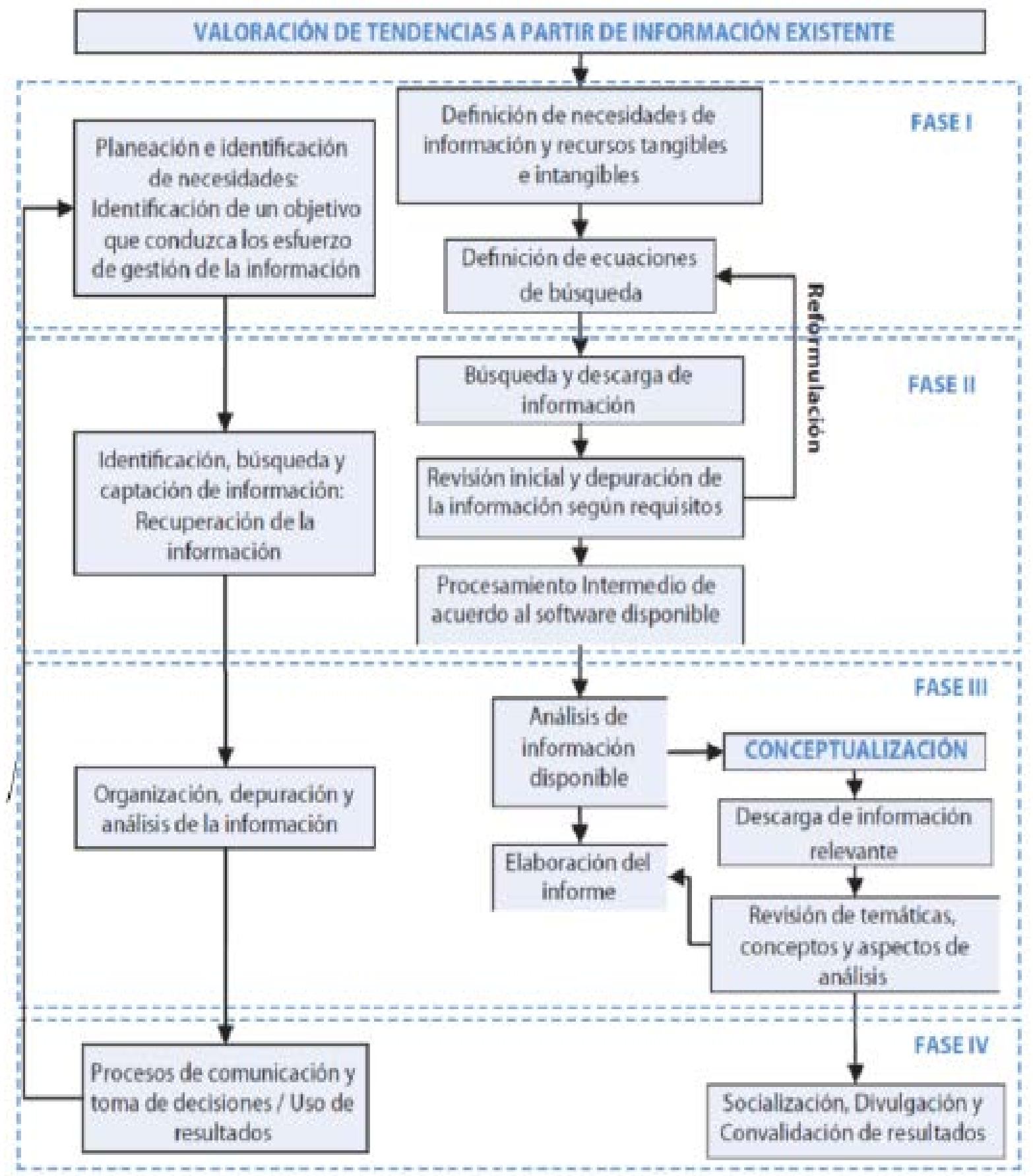

Nota: Esquema metodológico planteado por Castellanos Domínguez et al. (2011). 
Los objetivos de la investigación (figura 2) delimitaron el campo de búsqueda (necesidades de información). La etapa de búsqueda y descarga de información se adelantó utilizando la base de datos SCOPUS. En total, se probaron diecisiete ecuaciones de búsqueda en las que se relacionaron los términos: Responsabilidad
Social Universitaria, Responsabilidad Social en Universidades, Responsabilidad Social en la Educación Superior y evaluación de la Responsabilidad Social Universitaria. La tabla 1 muestra las ecuaciones de búsqueda planteadas y los resultados obtenidos.

Tabla 1

Búsqueda sistemática: resultados por ecuación.

\begin{tabular}{rlr}
\hline \multicolumn{1}{c}{ Ecuación de búsqueda } & Resultados \\
\hline 1 & Social accountability AND university & 156.412 \\
\hline 2 & Social responsibility AND university & 353.236 \\
\hline 3 & University social responsibility & 353.236 \\
\hline 4 & Social responsibility of universities & 353.236 \\
\hline 5 & Social responsibility in higher education & 90.611 \\
\hline 6 & Universitary AND social responsibility & 8 \\
\hline 7 & Universitary social AND responsibility & 8 \\
\hline 8 & Socially responsible AND university & 27.963 \\
\hline 9 & Socially responsible university & 27.963 \\
\hline 10 & "Socially responsible" AND university & 21.866 \\
\hline 11 & "Socially responsible university" & 149 \\
\hline 12 & "Socially responsible" AND "university" & 21.866 \\
\hline 13 & "Socially responsible" AND "higher education" & 2.399 \\
\hline 14 & "Socially responsible" AND "high education" & 2.399 \\
\hline 15 & Social AND responsibility AND measurement & 78.131 \\
\hline 16 & Social responsibility AND evaluation & 138.070 \\
\hline 17 & Social AND responsibility AND evaluation AND university & 119.165 \\
\hline & & 1.746 .718 \\
\hline
\end{tabular}

Las ecuaciones de búsqueda que arrojaron un mayor número de resultados fueron combinadas y filtradas, limitando los hallazgos según área temática y palabras clave. Del mismo modo, los documentos a revisar se restringieron a las categorías: articles, review, book, book chapter y undefined, derivados de investigaciones terminadas. Se excluyeron, además, un conjunto de palabras que podrían dirigir la búsqueda a campos como la educación básica, secundaria o el mundo empresarial. La tabla 2 presenta la ecuación de búsqueda final utilizada para refinar los resultados.

\section{Tabla 2}

Ecuación de búsqueda final

\section{Ecuación de búsqueda}

University Social Responsibility OR social responsibility of universities $O R$ social responsibility in higher education AND NOT school AND NOT corporate AND NOT industry AND NOT industries AND NOT health AND NOT enterprise
Áreas temáticas

\section{Palabras clave}

Social sciences; psychology; business, ma- Sin restricciones.

nagement and accounting; economics, econometrics and finance; engineering; multidisciplinary. 
En total, se listaron 556 publicaciones. Tales investigaciones fueron depuradas aplicando la técnica | Resultados de análisis de contenido (Berelson, 1952; Fernández, 2002; López-Noguero, 2002) al conjunto de títulos y resúmenes exportados a Microsoft Excel. Se utilizaron categorías tipo "materias o asuntos" en función de la Responsabilidad Social Universitaria, la Responsabilidad Social en Universidades, la Responsabilidad Social en Instituciones de Educación Superior, la Responsabilidad Social en la Educación Superior y la Evaluación de la Responsabilidad Social Universitaria, siguiendo lo planteado por Fernández (2002) y Piñuel Raigada (2002).

Entre los hallazgos más representativos se encontraron las investigaciones teóricas; los estudios de percepción, los estudios de caso, la socialización de prácticas de RSU y los intentos por hacer una medición/evaluación.

Dichos resultados guardan coherencia con la clasificación ofrecida por Duque y Cervantes-Cervantes (2019), quienes identificaron tres perspectivas ${ }^{1}$ en el estudio de la RSU: (1) medición de la RSU, (2) estratégico y (3) conexión RSU y sociedad.

A continuación, se presenta un análisis detallado de las publicaciones dirigidas a evaluar la RSU, con miras a establecer las dimensiones $y$ variables de medición empleadas a la fecha $y$ determinar posibles vacíos y campos de desarrollo.

Los avances en la evaluación de la RSU iniciaron con la propuesta del Banco Interamericano de Desarrollo (BID) (Vallaeys et al., 2009). En ella se crearon una serie de indicadores generales en un instrumento del que no se presentaron exámenes de validez o fiabilidad (BacaNeglia et al., 2017). Autores como Gallardo-Vázquez (2019), Aldeanueva Fernández y Arrabal Sánchez (2018), Gómez et al. (2018a), Da Silva Junior et al.(2018), Latif (2018), Baca-Neglia et al.(2017), Guijarro Jiménez et al (2016), Urdaneta, et al. (2016), López Vélez (2016), de la Calle Maldonado de Guevara et al. (2008), Nuñez Chicharro et al. (2015), Ramallo (2015), Garde Sánchez et al. (2013), Alba Hidalgo, et al. (2012), Casilla y Camacho (2012), Hernández y Saldarriaga (2009), Viteri-Moya et al. (2012), Aristimuño et al. (2011) así como redes internacionales entre las que se cuentan URSULA (URSULA, 2018), ORSALC (Grimaldo et al., 2018) y AUSJAL (2009) han hecho esfuerzos importantes por generar indicadores, escalas y sistemas de evaluación desde la óptica de la RSU y la sostenibilidad.

La tabla 3 presenta una descripción de las investigaciones mencionadas y el contexto de cada una, ordenadas de la más reciente a la más antigua.

Tabla 3

Evaluación de la RSU

\section{Publicación}

Escala de medida de responsabilidad social Escala de medida orientada al estudiante. en el contexto universitario: una triple vi- Evalúa: competencias transversales (RS y sión basada en competencias, formación y comportamiento ético), formación en RS y participación del estudiante (Gallardo-Váz- participación en actividades universitarias. quez, 2019).

Practicando la Responsabilidad Social Uni- Actitudes de públicos internos hacia prácti- Universidad privada de Puerto Rico. ${ }^{2}$ versitaria en el Caribe: Perspectivas de los cas de RS (375 estudiantes, 100 profesores y públicos internos (Gómez et al., 2018a).
110 administrativos): Campus responsable; educación profesional y ciudadana; gestión de conocimiento social; participación social. 


\section{Publicación}

Sustainability Indicators for the Management of Brazilian Higher Education Institutions (da Silva Junior et al., 2018).

The Development and Validation of Stakeholder-Based Scale for Measuring University Social Responsibility (USR) (Latif, 2018).

Propuesta de medición de la responsabilidad social universitaria (Baca-Neglia et al., 2017).

Propuesta de indicadores de la Responsabilidad Social Universitaria conforme a la guía G4 del GRI: el caso de la Universidad de Córdoba (Guijarro Jiménez et al., 2016).

Responsabilidad social en las universidades del municipio Maracaibo del estado Zulia (Urdaneta et al., 2016).

Propuesta de modelo de evaluación de la Innovación Social Universitaria Responsable (ISUR) (López Vélez, 2016).

Validación y medida de la responsabilidad social en la universidad (de la Calle Maldonado de Guevara et al., 2008).

La evaluación de la Responsabilidad Social Universitaria (Ramallo, 2015).

Online disclosure of university social responsibility: a comparative study of public and private US universities (Garde Sánchez et al., 2013).

Evaluación de la Responsabilidad Social Universitaria (Casilla \& Camacho, 2012).

Estrategias de Sostenibilidad y Responsabilidad Social en las Universidades Españolas: Una herramienta para su evaluación (Alba Hidalgo et al., 2012).

Índice integral para evaluar la responsabilidad social universitaria en Ecuador (Viteri-Moya et al., 2012).

La Responsabilidad Social Universitaria: Indicadores para su evaluación en Instituciones de Educación Superior (Aristimuño et al., 2011).

Gestión de la Responsabilidad Social Universitaria. Caso: Escuela de Ingeniería de Antioquia-EIA- (Hernández \& Saldarriaga, 2009).

Measuring social responsiveness of medical schools: A case study from Thailand (Yarmoshuk A.N.et al,1999).

\section{Descripción}

Propuesta de un conjunto de indicadores Brasil

de desempeño en RS y sostenibilidad aplicados a las IES Brasileñas.

Desarrollo y validación de escala de medi- No especificado. ${ }^{4}$

ción de la RSU compuesta de siete dimensiones. $^{3}$

Diseño de dos instrumentos (lista de cotejo Universidad San Martín de Porres (Perú).

y cuestionario) para analizar la gestión de

RSU (evaluación de fiabilidad, validez y capacidad diagnóstica).

Adaptación de los indicadores de la Guía G4 Universidad de Córdoba (UCO), España.

GRI a las universidades públicas españolas.

Propuesta de cinco instrumentos de me- Siete universidades públicas y privadas del dición. Incluye: políticas de RS, docencia, municipio de Maracaibo. gestión interna, investigación y extensión.

Modelo de evaluación de la ISUR ${ }^{5}$, com- No especificado.

puesto de seis dimensiones.

Estudio exploratorio: construcción y valida- Universidad Francisco de Vitoria, Madrid. ción de una escala de medida de la RS en estudiantes universitarios.

Propuesta de indicadores para la medición No especificado. de RSU.

Analiza: (1) la RS como elemento esencial Universidades públicas y privadas de EE. UU. de: rendición de cuentas, transparencia informativa y uso de Internet y (2) la RS como factor diferenciador.

Propuesta de evaluación de proyectos y/o No especificado. programas RSU. No incluye indicadores o mediciones. Identifica dimensiones de evaluación.

Aplicación de un conjunto de indicadores Universidades públicas españolas ${ }^{6}$. para evaluar el avance de las políticas de sostenibilidad de 31 universidades españolas.

Diseño de un índice integral de RSU: (1) Ecuador

funciones sustantivas y (2) perspectivas ambiental y comunicacional.

Determinación de indicadores de RSU para No especificado. un modelo Integral de Evaluación de IES.

Definición de RSU e indicadores de evalua- Escuela de Ingeniería de Antioquia-EIA-. ción.

Medición de la capacidad de respuesta social de las escuelas de medicina.

Escuela de Medicina de Chulalongkurn, Tailandia.
Las investigaciones centradas en la evaluación de la RSU muestran una marcada tendencia a seguir los principios de la teoría de los stakeholders (Baca-Neglia et al., 2017; Da Silva Junior et al., 2018; Gallardo-Vázquez, 2019; Gómez et al., 2018b; Latif, 2018; Urdaneta et al., 2016) y, por tanto, se amparan en definiciones de RSU afines a la gestión de impactos (Vallaeys \& Álvarez Rodríguez, 2019) y una perspectiva gerencial (Gaete Quezada, 2011).

Constantemente se hace uso de la guía del GRI (Da Silva Junior et al., 2018; Garde Sánchez et al., 2013; Guijarro Jiménez et al., 2016) y los aportes del Banco 
Interamericano de Desarrollo (BID) (Baca-Neglia et al., 2017; Gómez et al., 2018b; Urdaneta et al., 2016) para la generación de esquemas de evaluación que adaptan indicadores empresariales al contexto de la educación superior, creando nuevas categorías y subcategorías de medición que incluyen aspectos académicos -formación, investigaciónyproyección social-ydegestión universitaria.

La mayor parte de los trabajos revisados pasa por alto el análisis de los resultados de la gestión de RSU, limitando la evaluación a la existencia y divulgación de declaraciones de políticas de RS, prácticas, iniciativas o lineamientos que guíen el actuar de las IES, aspecto que resulta llamativo si se tienen en cuenta las perspectivas teóricas desde las cuales se han construido los instrumentos mencionados - la teoría de los stakeholders y la perspectiva gerencial- y la intencionalidad que debe perseguir cualquier proceso de evaluación.

La información es recolectada a través de la revisión

Tabla 4

Referentes de la evaluación de la RSU en Latinoamérica de informes de gestión y sostenibilidad, memorias de RS, publicaciones en páginas web institucionales y la aplicación de encuestas, cuestionarios y entrevistas a diferentes miembros de la comunidad, primordialmente, stakeholders internos (administrativos, docentes y estudiantes), utilizando para ello herramientas de evaluación cuantitativas y cualitativas.

En Latinoamérica se han dado algunos pasos hacia la medición/evaluación de la RSU basados en criterios similares a los ya mencionados. Las iniciativas de AUSJAL, URSULA, ORSALC y BID, sirvieron como referentes para la aplicación de modelos de evaluación en universidades latinoamericanas (Baca-Neglia et al., 2017; Gómez et al., 2018b; Urdaneta et al., 2016) al ofrecer una aproximación de carácter general que parte de la definición de ejes, metas o aspectos a evaluar y su relación con los impactos asociados a las funciones misionales de las IES. La tabla 4 presenta los principales referentes para la creación de instrumentos de evaluación de la RSU en Latinoamérica.

\begin{tabular}{|c|c|c|}
\hline Institución & Ejes/Dimensiones & Impactos \\
\hline BID (2009) & $\begin{array}{l}\text { Campus responsable; formación profesio- } \\
\text { nal y ciudadana; gestión social y del cono- } \\
\text { cimiento; participación social. }\end{array}$ & $\begin{array}{l}\text { Cognición, participación, organización y edu- } \\
\text { cación. }\end{array}$ \\
\hline AUSJAL (2014) & Políticas, procesos y resultados. ${ }^{7}$ & $\begin{array}{l}\text { Educativo, cognoscitivo, social, organizacio- } \\
\text { nal y ambiental. }\end{array}$ \\
\hline ORSALC (2018) & $\begin{array}{l}\text { Buen gobierno y transparencia; Clima la- } \\
\text { boral; medio ambiente y campus verde; } \\
\text { rehumanización; aprendizaje servicio; } \\
\text { vinculación y voluntariado; stakeholder; } \\
\text { educación para el desarrollo sostenible; } \\
\text { ambiente y cultura juvenil. }\end{array}$ & No definidos \\
\hline URSULA (2019) & $\begin{array}{l}\text { (1) Clima laboral y equidad; (2) campus sos- } \\
\text { tenible; (3) ética, transparencia e inclusión; } \\
\text { (4) aprendizaje servicio; (5) inclusión curri- } \\
\text { cular ODS; (6) mallas diseñadas con actores } \\
\text { externos; (7) inter y transdisciplinariedad; } \\
\text { (8) investigación en y con la comunidad; (9) } \\
\text { producción y difusión de conocimientos } \\
\text { útiles; (10) integración de la proyección so- } \\
\text { cial con formación e investigación; (11) pro- } \\
\text { yectos co-creados, duraderos y de impacto; } \\
\text { (12) participación en la agenda de desarro- } \\
\text { llo nacional e internacional. }\end{array}$ & $\begin{array}{l}\text { Participación social, gestión organizacional, } \\
\text { cognición y formación. }\end{array}$ \\
\hline
\end{tabular}


López Vélez (2016) desarrolla una propuesta de medición de la innovación social, un concepto asociado a la solución de problemas sociales, la generación de mejoras y la satisfacción de las necesidades de la sociedad de una manera más eficaz, eficiente

Tabla 5

Dimensiones y factores: modelo de evaluación ISUR y sostenible (López Vélez, 2016; Phills et al., 2008). Las dimensiones y factores que componen este modelo de evaluación se muestran en la tabla 5 .
Institución

D1. Curricular y pedagógica

D1. Curnicular y pedagógica

D2. Organizacional

D3. Investigación, desarrollo e innovación, I+D+I

D4. Relación con el entorno

D5. Ambiental

D6. Internacionalización

\section{Ejes/Dimensiones}

I. Formación del alumnado.

II. Desarrollo profesional para el personal universitario.

III. Respuesta educativa con equidad atendiendo a la diversidad.

I. Planificación, gestión y evaluación institucional.

II. Estructura organizacional.

III. Cultura organizacional.

I. Políticas institucionales de investigación.

II. Comunidad investigadora y redes de producción de conocimiento.

III. Gestión, transparencia y difusión de conocimiento.

IV. Fomento de la investigación en temas prioritarios.

I. Relación con la sociedad, proyectos, contribución al desarrollo del país.

II. Trabajos en red.

III. Incidencia en políticas públicas.

IV. Conocimiento académico popular.

I. Cultura ambiental.

II. Políticas y sistemas de gestión ambiental.

III. Formación en el área del medio ambiente.

IV. Investigación en el área de medio ambiente.

V. Acción en relación con el medio ambiente.

I. Políticas de fomento de internacionalización.

II. Trabajo de enfoque social en redes internacionales.

III. Movilidad académica internacional.

IV. Programas de formación conjunta.

Nota: La tabla resume las dimensiones y factores evaluados por López Vélez (2016).

La internacionalización aparece en este esquema como un complemento a la evaluación de las dimensiones curriculares, pedagógicas, organizacionales, investigativas, ambientales y de relacionamiento con el entorno, tras considerar que ésta enriquece la educación y la labor académica al estar abierta a la diferencia y la diversidad (Harris, 2008; López Vélez, 2016).

La sostenibilidad económica y/o financiera es otro de los factores que a menudo pasa inadvertido en las propuestas revisadas. Los trabajos de Guijarro Jiménez et al. (2016) y Da Silva Junior et al. (2018) se diferencian de los demás al incluir ítems para la evaluación de estos aspectos. A pesar de que el fin último de las IES no es la rentabilidad (Vallaeys, 2008), es necesario hacer seguimiento a sus finanzas con el ánimo de garantizar su supervivencia y crecimiento. Es por esto que resulta indispensable incluir indicadores que midan la estabilidad de corto y mediano plazo, con miras a garantizar el cumplimiento de las 
obligaciones adquiridas con los stakeholders y la sociedad.

Otros aspectos que aparecen en limitadas ocasiones son las certificaciones nacionales o internacionales de calidad universitaria (Da Silva Junior et al., 2018) u otras que, como las normas ISO dan cuenta de la calidad de los procesos de gestión (López Vélez, 2016) o la implementación de prácticas amigables con el medio ambiente.

Una evaluación integral de la RSU puede articularse con sistemas de gestión y aseguramiento de la calidad universitaria. Sus objetivos son similares en la medida en que promueve la mejora continua y el cumplimiento de los compromisos de la Universidad que, como se ha visto, en esencia son de carácter social.

Las dimensiones y factores generales de evaluación que pueden incluirse en un instrumento de evaluación de la RSU se resumen en la figura 3 .

\section{Figura 3}

Dimensiones y factores de evaluación RSU

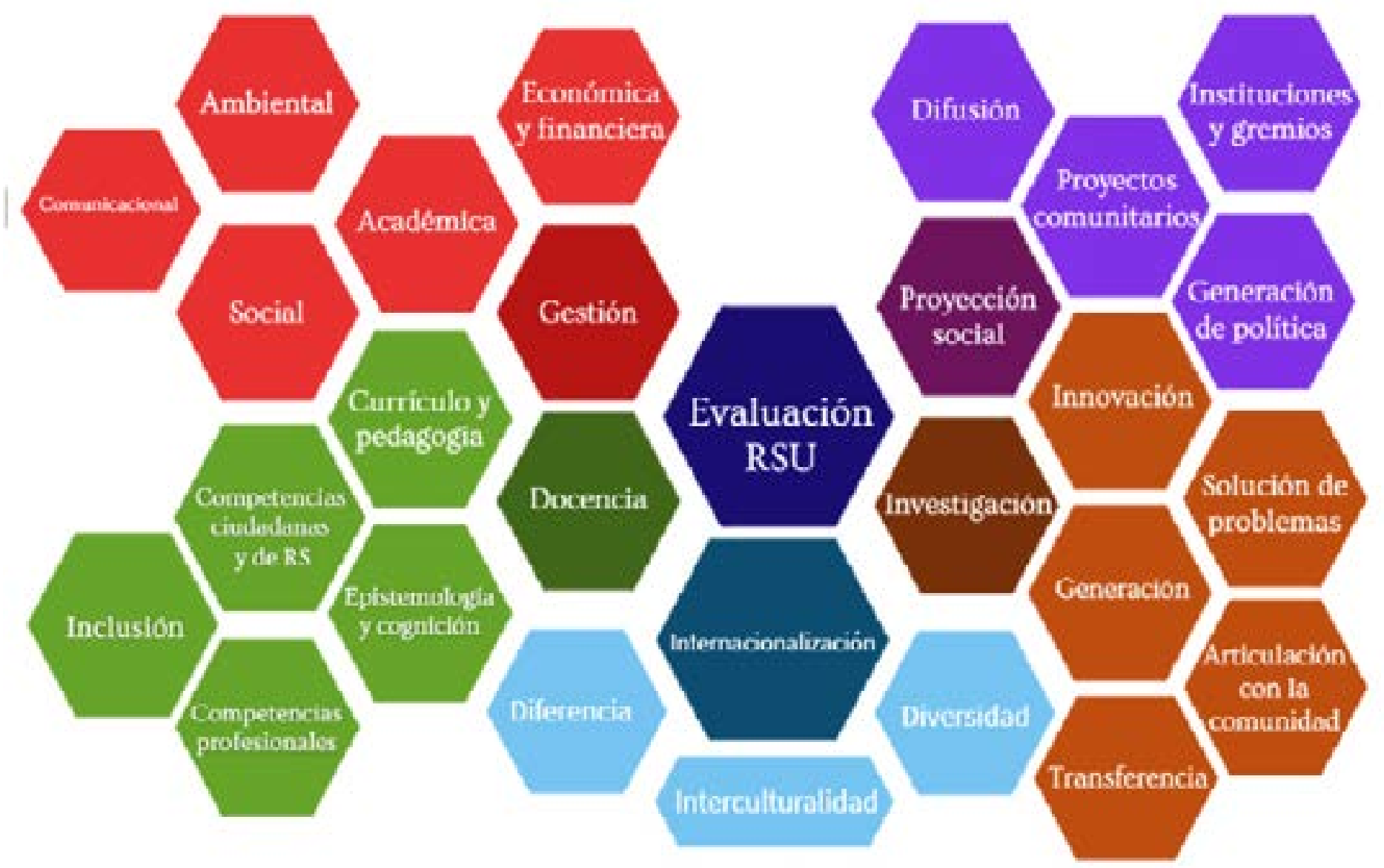


Se aprecia que el direccionamiento estratégico y la gestión universitaria deben integrar las dimensiones económica, social y ambiental, al igual que estrategias académicas y comunicacionales para el reporte de actividades y resultados de las funciones sustantivas de docencia, investigación, proyección social e internacionalización.

El proceso requiere de la recopilación de las percepciones de stakeholders externos e internos sobre el grado de cumplimiento de los principios, políticas y prácticas de RSU que guían la labor universitaria y la presentación de datos cualitativos y cuantitativos que den cuenta del cumplimiento de responsabilidades (1) operativas, (2) legales, (3) éticas, (4) comunitarias, (5) filantrópicas, (6) investigativas y (7) internas (Latif, 2018).

Con estos criterios se cubre la totalidad de las actividades desarrolladas por las IES en el marco de su operación normal, al tiempo que se exploran sus actuaciones bajo la mirada de la RSU.

\section{| Conclusiones}

La RSU se ha convertido, en las últimas décadas, en el paradigma reinante de la gestión universitaria. Evidencia de ello es el aumento de la producción científica a nivel mundial y el surgimiento de organismos internacionales promotores de su implementación y estudio. Al igual que otros sistemas de gestión, requiere del autodiagnóstico como punto de partida para la generación de esquemas administrativos dirigidos a asegurar la calidad en los procesos organizacionales y dar cumplimiento a la misión institucional.

La presente investigación se adelantó con el propósito de hacer una revisión de los instrumentos creados para evaluar la gestión de la RSU en IES de distintas características y contextos, con el ánimo de establecer las principales dimensiones de análisis y los posibles vacíos en su construcción. Así, se logró establecer como tendencia general la gran importancia dada a las percepciones de los stakeholders internos. Algunas de estas herramientas incluyen a egresados y padres de familia, ninguna tiene en cuenta a representantes del sector real o instituciones de apoyo. En su mayoría miden las apreciaciones de estudiantes, directivos, trabajadores y docentes sobre la implementación de principios, políticas y prácticas de RSU. En pocos casos se indaga sobre resultados.

Los instrumentos desarrollados se rigen por la teoría de los stakeholders; sin embargo, una evaluación surgida en el seno de la teoría de la legitimidad, que cuestione a comunidades, instituciones y sector empresarial acerca del rol que debe desempeñar la universidad en la sociedad actual y el impacto deseado de sus funciones sustantivas, permitiría conocer el grado de cumplimiento de las responsabilidades que los diferentes actores sociales les han conferido.

Las posturas institucionales y prácticas de gestión adelantadas frente a problemáticas como la desigualdad, la discriminación, la violencia de género, el racismo y la guerra no aparecen en los esquemas examinados, por tanto, pueden ser trabajados en profundidad en nuevas investigaciones. Los programas de inclusión y las iniciativas tendientes a aportar al logro de los Objetivos de Desarrollo Sostenible del Milenio (ODS), constituyen nuevos aspectos a considerar en los procesos de autodiagnóstico institucional.

$\mathrm{Al}$ igual que otras investigaciones de este tipo, la revisión sistemática desarrollada se encuentra limitada por los alcances de la metodología empleada. Para futuros estudios se sugiere ampliar la búsqueda incluyendoWeb of Science y otros metabuscadores, con el ánimo de obtener resultados que mejoren la comprensión de este fenómeno. 


\section{| Notas}

${ }^{1}$ Duque y Cervantes-Cervantes (2019) procesan una red de 260 nodos y 589 enlaces, empleando el criterio de puntuación más alta en los indicadores indegree (grado de entrada), betweenness (intermediación) y outdegree (grado de salida) para hacer su análisis. Como resultado, clasifican las investigaciones sobre RSU en tres grandes categorías: (a) documentos clásicos o hegemónicos (alto valor de Indegree y Outdegree de 0), (b) documentos actuales o recientes (alto valor Outdegree e Indegree de 0) y (c) documentos estructurales (alto valor betweenness).

${ }^{2}$ El autor no menciona el nombre de la Universidad objeto de estudio.

3 (1) Responsabilidades operativas, (2) responsabilidades de los actores internos, (3) responsabilidades legales, (4) responsabilidades éticas, (5) responsabilidades de investigación/ desarrollo, (6) responsabilidades filantrópicas y (7) compromiso con la comunidad.

${ }^{4}$ Aplica los principios generalmente aceptados para el diseño de instrumentos de Churchill (1979).

${ }^{5}$ Innovación Social Universitaria Responsable.

6 Las 31 universidades que participaron en el estudio representan el 40\% del sistema universitario español. 30 de las 31 universidades son de carácter público (Alba Hidalgo et al., 2012).

7 La políticas, procesos y resultados evalúan un total de veinticuatro ítems.

\section{| Referencias}

Alba Hidalgo, D., Barbeitos Alcántara, R., Barral Silva, M. T., Benayas del Álamo, J., Blanco Heras, D., Domenech Antúnez, X., Fernández Sánchez, I., Floresa i Botines, A., García Orenes, F., López Álvarez, N., \& Ysern
Comas, P. (2012). Estrategias de Sostenibilidad y Responsabilidad Social en las Universidades Españolas: Una herramienta para su evaluación. Profesorado. Revista de Currículum y Formación de Profesorado, 16(2), 59-75.

Aldeanueva Fernández, I., \& Arrabal Sánchez, G. (2018). La comunicación y medición de la Responsabilidad Social Universitaria: Redes sociales y propuesta de indicadores. Revista Digital de Investigación en Docencia Universitaria, 12(1), 121-136.

Amato, C. N., Buraschi, M., \& Peretti, M. F. (2016). Orientación de los empresarios de Córdoba-Argentina hacia la sustentabilidad y la responsabilidad social empresarial: identificación de variables asociadas a cada constructo. Contaduría y Administración, 61(1), 84-105. https://doi.org/h6fq

Aristimuño, M., Rodríguez, C., \& Guaita, W. (3-5 de noviembre de 2011). La Responsabilidad Social Universitaria: Indicadores para su evaluación en Instituciones de Educación Superior. [Ponencia]. Ninth LACCEI Latin American and Caribbean Conference, Engineering for a Smart Planet, Innovation, Information Technology and Computational Tools for Sustainable Development, Medellín Colombia. https://cutt.ly/6YRDGKz

Asociación de Universidades confiadas a la Compañía de Jesús en América Latina [AUSJAL]-Red, R. (2009). Políticas y sistema de autoevaluación y gestión de la responsabilidad social universitaria en AUSJAL. Alejandría Editorial. https://cutt.ly/XZhlX3R

Baca-Neglia, H. Z., Rondán-Cataluña, F. J., \& García-Del-Junco, J. (2017). Propuesta de medición de la responsabilidad social universitaria. Espacios, 38(43), 1-37.

Berelson, B. (1952). Content analysis in communication research. Hafner.

Caro González, F. J., \& Castellanos Verdugo, M. (2007). Propuesta de una escala de medición de la Responsabilidad Social Corporativa (RSC) en la actividad turística. Conocimiento, Innovación y Emprendedores: Camino al Futuro, 2621-2632. https://cutt.ly/hYRD065

Casilla, D., \& Camacho, H. (2012). Evaluación de la respon- 
sabilidad social universitaria. Opción, 28(69), 452-465.

Castellanos Domínguez, O. F., Fúquene Montañez, A. M., \& Ramírez Martínez, D. C. (2011). Análisis de tendencias: de la información hacia la innovación. Universidad Nacional de Colombia.

Churchill, G. A. (1979). A Paradigm for Developing Better Measures of Marketing Constructs. Journal of Marketing Research, 16(1), 64. https://doi.org/dd8dpw

Comisión de las Comunidades Europeas. (2002). Libro verde. Fomentar un marco europeo para la responsabilidad social de las empresas. https://cutt.ly/7YRFoqa

Coppa, C. R. (2011). Responsabilidad Social Universitaria: nuevo paradigma en la educación superior. Anuario de La Facultad de Ciencias Económicas Del Rosario, 7, 85-98.

Correa, M., Flynn, S., \& Amit, A. (2004). Responsabilidad social corporativa en América Latina: una visión empresarial. In CEPAL - SERIE Medio Ambiente y Desarrollo.

Crespo Razeg, F. (2010). Entre el concepto y la práctica: responsabilidad social empresarial. Estudios Gerenciales, 26(117), 119-130. https://doi.org/f2mqv2

Da Silva Junior, A., Martins-Silva, P. de O., Vasconcelos de Araújo, K. C., da Silva, V. C., Ramos de Melo, M., \& Ramos Dumer, M. C. (2018). Sustainability indicators for the management of Brazilian higher education institutions. BAR - Brazilian Administration Review, 15(3), 1-22. https://doi.org/h6f5

De la Calle Maldonado de Guevara, C., García Ramos, J. M., Giménez Armentia, P., \& Ortega de la Fuente, M. (2008). Validación y medida de la responsabilidad social en la universidad. Revista Complutense de Educación, 19(2), 385-404.

De la Cuesta González, M. (2004). El porqué de la responsabilidad social corporativa. Boletín Económico de ICE, 2913, 45-58.

De la Cuesta González, M., \& Valor Martínez, C. (2003). Responsabilidad social de la empresa Concepto, medición y desarrollo en España. Boletín ICE Económico, 2755, 7-20.

De Sousa Santos, B. (2007). La Universidad En El Siglo XXI.
Para una reforma democrática y emancipatoria de la Universidad. In Educación para otro mundo posible. CIDES-IMSA, Asdi y Plural Editores. https://doi.org/ $\underline{\text { h6f6 }}$

Duque, P., \& Cervantes-Cervantes, L.-S. (2019). Responsabilidad Social Universitaria: una revisión sistemática y análisis bibliométrico. Estudios Gerenciales, 35(153), 451-464. https://doi.org/h6f7

Eberhard-Harribey, L. (2006). Corporate social responsibility as a new paradigm in the European policy: how CSR comes to legitimate the European regulation process. Corporate Governance, 6(4), 358.

Fernández, F. (2002). El análisis de contenido como ayuda metodológica para la investigación. Revista de Ciencias Sociales (San José), 96, 35-53.

Ferreras-Fernández, T., Martín-Rodero, H., García-Peñalvo, Francisco, J., \& Merlo-Vega, José, A. (2016). The systematic review of literature in LIS: An approach. ACM International Conference Proceeding Series, 02-04-Nove, 291-298. https://doi.org/gjkjm5

Fink, A. (1998). Conducting literature research reviews: from paper to the Internet. Sage Publications.

Gaete Quezada, R. (2016a). La responsabilidad social universitaria en la identidad corporativa de las universidades chilenas. un análisis de contenido. Documentos y Aportes en Administración Pública y Gestión Estatal, 16(26), 43-74.

Gaete Quezada, R. (2016b). Percepción de los dirigentes estudiantiles universitarios sobre la responsabilidad social universitaria. OBETS. Revista de Ciencias Sociales, 11(2), 461-485. https://doi.org/h6f8

Gallardo-Vázquez, D. A. (2019). Escala de medida de responsabilidad social en el contexto universitario: una triple visión basada en competencias, formación y participación del estudiante. Estudios Gerenciales, 35(151), 159-177. https://doi.org/h6f9

García-Peñalvo, F. J. (2017). Revisión sistemática de literatura en los Trabajos de Final de Máster y en las Tesis Doctorales. Grial, 95.

Garde Sánchez, R., Rodríguez Bolívar, M. P., \& López-Her- 
nández, A. M. (2013). Online disclosure of university social responsibility: a comparative study of public and private US universities. Environmental Education Research, 19(6), 709-746.

Gómez, L. M., Alvarado Naveira, Y., \& Pujols Bernabel, A. (2018a). Implementing University Social Responsibility in the Caribbean: Perspectives of Internal Stakeholders. Revista Digital de Investigación en Docencia Universitaria, 12(1), 101-120. https://doi.org/h6gb

Gómez, L. M., Alvarado Naveira, Y., \& Pujols Bernabel, A. (2018b). Practicando la Responsabilidad Social Universitaria en el Caribe: Perspectivas de los públicos internos. Revista Digital de Investigación en Docencia Universitaria, 12(1), 101-120.

Grimaldo, H., Fierro, A., Didriksson, A., Riego, I., Martí Noguera, J., Zavaleta, M., Licandro, O., Guadarrama, P., \& Fiorino, V. M. (2018). El rol de la educación superior de cara a los desafíos sociales de América Latina y el Caribe. Editorial Universidad Nacional de Córdoba. https:// cutt.ly/4YRFZtQ

Guijarro Jiménez, C., Gomera Martínez, A., \& Antúnez López, M. (2016). Propuesta de indicadores de la Responsabilidad Social Universitaria conforme a la guía G4 del GRI: el caso de la Universidad de Córdoba. CIRIEC-España, Revista de Economía Pública, Social y Cooperativa, 87, 103-137.

Harris, S. (2008). La dimensión internacional de la universidad: entre el modelo económico y el cultural. Estudios Sobre Educación, 15, 87-98.

Hart, A., \& Northmore, S. (2010). Auditing and evaluating University-Community Engagement: Lessons from a UK Case Study. Higher Education Quarterly, 65(1), 34-58.

Hernández, R. D., \& Saldarriaga, A. (2009). Gestión de la Responsabilidad Socuial Universitaria. Caso: Escuela de Ingeniería de Antioquia-EIA. Dyna, 76(159), 237248.

Jiménez, M., de Ferrari, J., Delpiano, C., \& Andrade, L. (2004). Observando la responsabilidad social universitaria. Universidad Construye País.
Latif, K. F. (2018). The Development and Validation of Stakeholder-Based Scale for Measuring University Social Responsibility (USR). Social Indicators Research, 140(2), 511-547. https://doi.org/gfksvk

Logsdon, J. M. (2004). Global Business Citizenship: Applications to Environmental Issues. Business and Society Review, 109(1), 67.

Londoño Franco, I. C. (2013). Responsabilidad Social Universitaria - una estrategia de gestión para la educación superior. Sinapsis, 5(5), 137-151.

López Vélez, A. L. (2016). Propuesta de modelo de evaluación de la Innovación Social Universitaria Responsable (ISUR). Estudios Sobre Educacion, 30, 71-93. https:// doi.org/h6gc

López-Noguero, F. (2002). El análisis de contenido como método de investigación. XXI. Revista de Educación, 4, 167-180.

Lozano, R. (2006). A tool for a Graphical Assessment of Sustainability in Universities (Gasu). Journal of Cleaner Production, 14(9-11), 963-972.

Maier, H. R. (2013). What constitutes a good literature review and why does its quality matter? Environmental Modelling \& Software, 43, 3-4.

Martínez García de Leaniz, P., \& Rodríguez del Bosque Rodríguez, I. (2013). Revisión teórica del concepto y estrategias de medición de la Responsabilidad Social Corporativa. Prisma Social, 11, 321-350.

Martinez, M., \& Soza, C. (2012). La responsabilidad social de las empresas: orígenes , aproximaciones conceptuales y estrategias. La Responsabilidad Social de La Empresa, 97-110.

Nieto Antolín, M., \& Fernández Gago, R. (2004). Responsabilidad social corporativa: la última innovación en management. Universia Business Review, 28-38.

Nuñez Chicharro, M., Alonso Carrillo, I., \& Pontones Rosa, C. (2015). Responsabilidad Social Universitaria: estudio empírico sobre la fiabilidad de un conjunto de indicadores de Gobierno Corporativo. INNOVAR. Revista de Ciencias Administrativas y Sociales, 25(58), 91-104. https://doi.org/h6gd.CITACI 
Phills, J., Jr., Deiglmeier, K., \& Miller, D. (2008). Rediscovering social innovation. Stanford Social Innovation Review, 6(2), 34-43.

Piñuel Raigada, J.L. (2002). Epistemología, metodología y técnicas del análisis de contenido. Sociolinguistic Studies, 3(1), 1-42. https://doi.org/cz7vk3

Ramallo, M. (2015). La evaluación de la Responsabilidad Social Universitaria. Debate Universitario, 4(7), 25-38.

Raufflet, E., Lozano, J. F., Barrera, E., \& García de la Torre, C. (2012). Responsabilidad social empresarial. Pearson

Rodríguez Fernández, J. M. (2010). Responsabilidad social universitaria: del discurso simbólico a los desafíos reales. En M. de la Cuesta González et al. (Ed.), Responsabilidad Social Universitaria (pp. 3-24). Netbiblo.

Sáenz, A. (2001). Leer e interpretar una revisión sistemática. Boletín de La Sociedad de Pediatría de Asturias, Cantabria, Castilla y León, 41(177), 215-221.

Torres Castaño, A. G., \& Sánchez Vasquez, L. M. (2014). La Responsabilidad Social Universitaria desde su fundamentación teórica. Libre Empresa, 11(1), 69-105. https://doi.org/h6gf

Organización de las Naciones Unidas para la Educación, la Ciencia y la Cultura [UNESCO]. (1998). Declaración mundial sobre la educación superior en el siglo XXI: visión y acción. https://cutt.ly/xYRGvGQ

Organización de las Naciones Unidas para la Educación, la Ciencia y la Cultura [UNESCO]. (2009). Conferencia Mundial sobre la Educación Superior - 2009: La nueva dinámica de la educación superior y la investigación para el cambio social y el desarrollo. https://cutt.ly/jYRGOIL

Unión de Responsabilidad Social Universitaria Latinoamericana [URSULA]. (2018). Resultados investigación continental URSULA: Estado del arte de la RSU en América Latina 2018. https://cutt.ly/PYRG067

Urdaneta, M., Cova, M. L., Chirinos, A., \& Gonzalez, X. (2016). Responsabilidad social en las universidades del municipio Maracaibo del estado Zulia. Revista Venezolana de Gerencia, 73, 65-85.

Vallaeys, F. (2008). ¿Qué es la responsabilidad social universitaria? https://cutt.ly/xYRHtqx
Vallaeys, F. (2014). La responsabilidad social universitaria: un nuevo modelo universitario contra la mercantilización. Revista Iberoamericana de Educación Superior, 5(12), 105-117.

Vallaeys, F., \& Álvarez Rodríguez, J. (2019). Hacia una definición latinoamericana de Responsabilidad Social Universitaria. Aproximación a las preferencias conceptuales de los universitarios. Educación XX1, 22(1), 93-116. https://doi.org/h6gg

Vallaeys, F., De la Cruz Ayuso, C., \& Sasia, P. M. (2009). Responsabilidad social universitaria. Manual de primeros pasos. McGraw-Hill Interamericana.

Villar, J. (2009). Responsabilidad social universitaria: nuevos paradigmas para una educación liberadora y humanizadora de las personas y las sociedades. Responsabilidad Social, 4, 27-37.

Viteri-Moya, J., Jácome-Villacres, M., \& Medina-León, A. (2012). Índice integral para evaluar la responsabilidad social universitaria en Ecuador. Ingeniería Industrial, 33(3), 295-306.

Votaw, D. (1972). Genius Became Rare: A Comment on the Doctrine of Social Responsibility. California Management Review, 15(2), 25-31.

World Business Council for Sustainable Development [WBCSD] \& United Nations Environment Programme Finance Initiative [UNEP FI]. (2004). Generation lost: young financial analysts and environmental, social and governance issues. WBCSD.

Wheeler, D., Colbert, B., \& Freeman, R. E. (2003). Focusing on Value : Reconciling Corporate Social Responsibility, Sustainability and a Stakeholder Approach in a Network World. Journal of General Management, 28(3).

Zárate Rueda, R., \& García Rincón, S. C. (2014). La cultura socialmente responsable de la UIS: una perspectiva desde el ámbito educativo y social. Revista Encuentros, 12(2), 105-120. https://doi.org/h6gh 